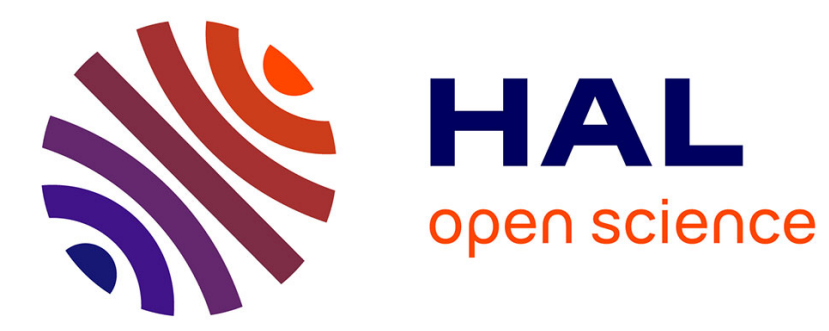

\title{
Pour une Épistémologie de la Controverse Historiographique
}

Jochen Hoock, Wolfgang Kaiser

\section{To cite this version:}

Jochen Hoock, Wolfgang Kaiser. Pour une Épistémologie de la Controverse Historiographique. Revue de Synthèse, 2009, 130 (4), pp.679-695. 10.1007/s11873-009-0097-0 . hal-00545753

\section{HAL Id: hal-00545753 \\ https://hal.science/hal-00545753}

Submitted on 12 Dec 2010

HAL is a multi-disciplinary open access archive for the deposit and dissemination of scientific research documents, whether they are published or not. The documents may come from teaching and research institutions in France or abroad, or from public or private research centers.
L'archive ouverte pluridisciplinaire HAL, est destinée au dépôt et à la diffusion de documents scientifiques de niveau recherche, publiés ou non, émanant des établissements d'enseignement et de recherche français ou étrangers, des laboratoires publics ou privés. 


\title{
POUR UNE ÉPISTÉMOLOGIE DE LA CONTROVERSE HISTORIOGRAPHIQUE À propos des débats d'outre-Rhin
}

\author{
Jochen Hoock et Wolfgang KaISER*
}

RÉSUMÉ: Les grandes controverses historiographiques se sont généralement déroulées sur plusieurs fronts. L'article essaie de replacer les débats actuels dans le contexte et l'horizon de différentes expériences de recherche afin de dégager leur apport théorique et empirique. En envisageant surtout les rapports controversés entre histoire sociale et anthropologie historique, il cherche à dégager les récurrences qui le lient à d'autres débats dont les moments forts autour de 1800 et de 1900 reflètent des crises aux connotations politiques mais aussi aux conséquences épistémologiques évidentes.

Mots-CLÉs : théorie de l'histoire, histoire globale, histoire universelle.

\section{FÜR EINE EPISTEMOLOGIE HISTORISCHER KONTROVERSE \\ Zu den aktuellen Auseinandersetzungen jenseits des Rheins}

ZuSAMMENFASSUNG:Die großen historiographischen Auseinandersetzungen verliefen in der Regel zugleich an verschiedenen Fronten. Der Artikel versucht die gegenwärtigen Debatten im Zusammenhang und im Horizont der unterschiedlichen Forschungserfahrungen ins Auge zu fassen, um ihren theoretischen und empirischen Ertrag deutlich zu machen. Indem er sich vornehmlich auf das problematische Verhältnis von Gesellschaftsgeschichte und historischer Anthropologie beschränkt, geht er zugleich den sich wiederholenden Motiven nach, die an die Krisen um 1800 und 1900 und deren epistemologische Folgen erinnern.

STICHWÖRTER: Theorie der Geschichte, Partikulare Geschichte, Allgemeine Geschichte.

ON THE EPISTEMOLOGICAL IMPACT OF HISTORICAL DEBATES

Reconsidering the actual controversies between german historians

ABSTRACT: The great historical debates were generally fought on different fields at the same time. The present article tries to analyze the actual debates in the context and perspective of different research experiences, aiming to highlight their theoretical and empirical results. In focussing on the controversies about the history of society and historical anthropology it questions in the same time the recurrencies recalling the crisis at the end of the $18^{\text {th }}$ and the beginning of $20^{\text {th }}$ century and their epistemological consequences.

KEYWORDS: theory of history, global history, universal history.

* Jochen Hoock, né en 1939, est professeur émérite d'histoire moderne et contemporaine de l'université de Paris 7, Denis Diderot (2, place Jussieu, 75005 Paris; jochen.hoock@wanadoo.fr). Ses recherches portent sur l'histoire de la praxéologie économique et la théorie de l'histoire. Il a notamment publié, en collaboration avec Pierre Jeannin et Wolfgang Kaiser, Ars Mercatoria. Manuels et traité à l'usage des marchands, 1470-1700 (Paderborn, Schöningh, 1991-2001, 3 vol.).

Wolfgang Kaiser, né en 1951, est professeur d'histoire moderne à l'université de Paris 1 (Centre de recherches d'histoire moderne, 17, rue de la Sorbonne, 75005 Paris; wolfgang.kaiser@univ-paris1. fr) et directeur d'études à l'École des hautes études en sciences sociales. Ses recherches actuelles portent sur les échanges en Méditerranée à l'époque moderne. Il a notamment dirigé Le Commerce des captifs. Les intermédiaires dans l'échange et le rachat des prisonniers en Méditerranée, $X V^{e}-X V I I^{e}$ siècles (Rome, École française de Rome, 2008). 
$\mathrm{H}^{\mathrm{a}}$ ans-Ulrich Wehler a revendiqué pour l'historiographie allemande cinq débats de fond qui ont marqué son évolution et redéfini ses rapports avec son environnement politique, social et culturel ${ }^{1}$. Le premier serait celui qui opposa les historiens des Lumières et l'historisme naissant; le deuxième, le débat provoqué par l'attaque de Johann Gustav Droysen contre le scientisme naturaliste de Henry Thomas Buckle suivi par la controverse autour de l'œuvre de Karl Lamprecht; le quatrième et le cinquième, les débats théoriques des années 1960-1970 provoquant un tournant analytique dans le domaine de l'histoire sociale mis en cause, depuis, par l'irruption des multiples approches en histoire culturelle.

Si ce rapprochement s'avère pertinent c'est essentiellement par le fait que ces débats se sont déroulés sur de multiples fronts et ne se laissent guère réduire à un seul enjeu théorique ou méthodologique. Ce qui frappe surtout ici, c'est plutôt la récurrence de certains motifs dont la lutte sur deux fronts de Droysen, engagé autant contre Ranke que contre Buckle, pourrait servir de modèle, si les zones de fractures ne paraissaient pas encore beaucoup plus complexes et marquées par une mémoire disciplinaire qui fait tout pour brouiller encore un peu plus les cartes. Opter pour une analyse en termes de dynamique sociale et culturelle est de ce fait une des options plausibles choisie par Christoph Conrad dans une analyse très informée des tournants qui dépasse d'ailleurs le seul cas allemand ${ }^{2}$. Se situant, non sans ironie, dans une perspective mannheimienne

Gans-Ulrich Wehler hat für die deutsche Geschichtsschreibung fünf Grundsatzde-

1 batten ausgemacht, die ihre Entwicklung geprägt und ihre politische, soziale und kulturelle Position neu bestimmt haben ${ }^{1}$. In der ersten standen sich die Aufklärungshistoriker und die Vertreter des aufkommenden Historismus gegenüber. Die zweite wurde ausgelöst durch den Angriff Johann Gustav Droysens auf den naturwissenschaftlichen Szientismus Henry Thomas Buckles, gefolgt vom sogenannten Lamprecht-Streit (um den kulturgeschichtlichen Ansatz von Karl Lamprecht). Die vierte und fünfte seien die theoretischen Debatten der 1960er und 1970er Jahre gewesen, die eine analytische Wende in der Sozialgeschichte ausgelöst hätten, die dann durch verschiedene kulturgeschichtliche Ansätze grundsätzlich in Frage gestellt worden sei.

Ein solcher konzentrierender Rückblick scheint triftig, weil diese Kontroversen sich an mehreren Fronten abgespielt haben und sich kaum auf ein theoretisches oder methodisches Konfliktthema beschränken lassen. Erstaunlich ist vor allem die Wiederkehr bestimmter (argumentativer) Motive, für die der Zweifrontenkampf Droysens sowohl gegen Ranke wie gegen Buckle gleichsam ein Modell bilden könnte, wären die Bruchlinien nicht noch viel komplizierter gewesen, geprägt durch ein fachspezifisches Gedächtnis, das sie noch mehr verwischt. Die diesen Debatten zugrunde liegende soziale und kulturelle Dynamik zu untersuchen, ist deshalb eine plausible Option, für die sich Christoph Conrad in einer empirisch gesättigten Analyse der historiographischen Wenden entschieden hat - die im übrigen weit über den deutschen Fall hinausgeht ${ }^{2}$. Seine Untersuchung stellt sich, leicht ironisch, in eine wissenssoziologische Perspektive

1. WeHLER, 1997, p. 351.

2. CONRAD, 2006. 
ou bourdieusienne, elle fait de la controverse un enjeu servant à sa propre reproduction en ouvrant un champ de l'analyse socio-culturelle qui occupe désormais une place de choix dans la communication universitaire ${ }^{3}$.

Ramener le débat à une analyse critique des principes, des hypothèses et des résultats des courants dont se nourrissent les controverses est l'alternative dont témoignent la plupart des débats en cause. Les « résultats » du Lamprecht-Streit se mesurent autant en termes d'échec quand il s'agit du renouvellement de l'histoire médiévale qu'en termes de succès quand on envisage le développement de l'histoire régionale et de ses instruments, l'ouverture de l'éventail de sources que la controverse a provoquée. L'apparente circularité de l'argument ne doit pas cacher le fait que toute controverse est aussi l'expression du milieu où elle est née et dont la réceptivité n'est pas le moindre moteur de son développement. La controverse accorde à l'adversaire, souvent avec un mélange d'envie et de répulsion, d'avoir désigné un enjeu qui vaut la peine d'être envisagé. C'est en cela que réside en grande partie sa dimension à proprement parler épistémologique.

\section{Entre histoire sociale et anthropologie historique}

Ce qui a caractérisé le débat outre-Rhin dès le début des années 1960 est un haut degré de réflexivité auquel se mêlait une forte conscience des dangers et de la responsabilité

im Sinne Karl Mannheims oder Pierre Bourdieus und macht aus der Kontroverse ein sich selbst reproduzierendes Phänomen. Damit eröffnet sich ein Gebiet sozio-kultureller Analysen, die mittlerweile einen prominenten Platz in der universitären Kommunikation einnehmen ${ }^{3}$.

Ein alternatives Vorgehen bestünde darin, die Debatte auf eine kritische Analyse der Grundlagen, Hypothesen und Ergebnisse der Strömungen zurückzuführen, aus denen sich die Kontroversen speisen, wie sich an der Mehrzahl der hier in Rede stehenden Debatten belegen läßt. Die «Ergebnisse 》 des Lamprecht-Streits lassen sich als Niederlage deuten, soweit es um die Erneuerung der Mediävistik ging, als Erfolg jedoch hinsichtlich der Entwicklung der Regionalgeschichte und ihrer Instrumente, oder hinsichtlich der Erweiterung des Spektrums möglicher Quellen, welche die Kontroverse bewirkt hat. Die vermeintliche Zirkularität des Arguments sollte nicht darüber hinweg täuschen, daß die Kontroverse auch Ausdruck des Milieus ist, in dem sie entsteht: dessen Empfänglichkeit ist ein wichtiger Motor ihrer Entfaltung. Die Kontroverse gesteht dem Gegner zu, häufig in einer Mischung aus Neid und Abwehr, das Kernproblem bezeichnet zu haben, um das es sich zu streiten lohnt. Darin vor allem liegt ihre epistemologische Dimension.

Zwischen Sozialgeschichte und historischer Anthropologie

Die deutsche Debatte seit Anfang der 1960er Jahre zeichnet ein hoher Grad von Selbstreflexion aus, verbunden mit einem starken Bewußtsein der Gefahren und der

3. Voir CONRAD, 2006, p. 157, à propos des nombreux groupes de recherches concernant les performative turns dans le domaine du savoir culturel. 
de l'engagement scientifique ${ }^{4}$. Surdéterminé par le passé nazi, il ne pouvait qu'être un débat intergénérationnel où l' " intérêt pour l'histoire » devenait autant une interrogation sur le présent ${ }^{5}$. En 1957, la création du Arbeitskreis für moderne Sozialgeschichte à Heidelberg réunissait des courants extrêmement divers allant de la Volksgeschichte à l'histoire ouvrière et donnait naissance à une nouvelle histoire sociale qui allait devenir dans les deux décennies suivantes un des courants les plus novateurs de l'historiographie allemande ${ }^{6}$.

La vitalité du courant devait beaucoup à l'hétérogénéité de ses composantes que l'expression neutre d'une " histoire des structures » (Strukturgeschichte) ne pouvait occulter. Quand Theodor Schieder et Reinhard Wittram convoquèrent à partir de 1975 un groupe de travail sur la «Théorie de l'histoire » qui se réunira par la suite régulièrement sous la responsabilité de Rudolf Vierhaus, la reconstruction de la discipline avait pris entre temps le caractère d'une véritable ouverture ${ }^{7}$. Dix ans plus tôt, Theodor Schieder avait publié une introduction à l'histoire sous le titre Geschichte als Wissenschaft qui mêlait les références à l'anthropologie philosophique à celles de l'ethnographie anglo-américaine et des divers courants de la sociologie historique ${ }^{8}$. $\mathrm{Au}$ même moment paraissaient en langue allemande les travaux d'historiens comme

Verantwortung des wissenschaftlichen Engagements ${ }^{4}$. Überdeterminiert durch die Nazivergangenheit, mußte sie eine Debatte zwischen den Generationen werden, in der das "Interesse an der Geschichte » zugleich zum Befragen der Gegenwart wurde". Die Gründung des Arbeitskreises für moderne Sozialgeschichte in Heidelberg führte äußerst unterschiedliche Strömungen zusammen, die von der Volksgeschichte zur Klassengeschichte reichten. Daraus entstand eine neue Sozialgeschichte, die in den folgenden beiden Jahrzehnten eine der innovativsten Richtung der deutschen Geschichtswissenschaft werden sollte ${ }^{6}$.

Die Vitalität dieser Strömung verdankte sich sehr stark der Heterogeneität ihrer Bestandteile, ein Sachverhalt, den der neutrale Begriff "Strukturgeschichte 》 nicht verdecken konnte. Als Theodor Schieder und Reinhard Wittram 1975 eine Arbeitsgruppe zur "Theorie der Geschichte » bildeten, die später regelmäßig unter der Leitung von Rudolf Vierhaus zusammenkam, war aus dem Wiederaufbau des Fachs eine wirkliche Öffnung und Erweiterung geworden ${ }^{7}$. Zehn Jahre zuvor hatte Theodor Schieder eine Einführung in die Geschichte unter dem Titel Geschichte als Wissenschaft verfaßt, in der die Bezüge auf die philosophische Anthropologie neben jenen auf die anglo-amerikanische Ethnographie und auf die verschiedenen Richtungen der historischen Soziologie standen ${ }^{8}$. Gleichzeitig erschienen in deutscher Sprache die Arbeiten von Historikern wie Franz Neumann, Ernst Fraenkel und Hans Rosenberg, die 1933 Deutschland hatten verlassen müssen. 1970, zum Zeitpunkt einer

4. Voir à ce propos les analyses de Schulze, 1989, passim; LePENIES, 1990.

5. Das Interesse an der Geschichte fut le titre d'un ouvrage dans lequel Reinhard Wittram rendait compte, en 1958, de ses activités d'historien sous le III' Reich. Voir Schulze et OeXLe, éd., 1999.

6. Kоска, 2002.

7. Voir la collection Theorie der Geschichte, 1977-1990.

8. SCHIEDER, 1965. 
Franz Neumann, Ernst Fraenkel et Hans Rosenberg qui avaient dû quitter l'Allemagne en 1933. En 1970, au moment d'une expansion sans précédent du système universitaire, la nouvelle histoire sociale présentait une programmatique définissant un champ de recherche qui semblait justifier la création d'une faculté modèle pour la «science historique » : ce sera Bielefeld, qui au cours des deux décennies suivantes allait être identifiée à la nouvelle histoire sociale tout court.

A posteriori cette identification semble plus révélatrice des controverses des années 1980 et de leur force relative que de l'unité supposée d'une "école » qui très tôt a fait preuve d'une forte capacité de différenciation. L'histoire sociale, telle que la définissaient les principaux acteurs au début des années 1970, n'était pas un des secteurs de l'histoire mais bien plus une approche globalisante envisageant les faits politiques, économiques et culturels sous l'angle de la société ${ }^{9}$. La même observation vaut pour la revue Geschichte und Gesellschaft, créée en 1975, dont le programme était plus marqué par un sens développé de la polémique ouverte que par une approche étroitement sociologique comme pouvait le suggérer le sous-titre Zeitschrift für historische Sozialwissenschaft. Dans l'ensemble, le renouveau des années 1970 s'inscrivait dans un champ référentiel aux connotations parfois lointaines comme la dénomination généralisante des chaires d'histoire de l'université de Bielefeld, qui rappelait pour beaucoup d'observateurs celle créée en 1923 sous l'intitulé «Théorie de la société et science

beispiellosen Ausweitung des Universitätssystems, bot die neue Sozialgeschichte ein Programm, das ein Forschungsfeld definierte, welches die Schaffung einer Modellfakultät für die "Geschichtswissenschaft" zu rechtfertigen schien. Diese Fakultät wurde Bielefeld, die in den nächsten zwei Jahrzehnten einfach mit der neuen Sozialgeschichte identifiziert werden sollte.

Im Nachhinein erscheint diese Identifikation eher Aufschluß zu geben über die Kontroversen der 1980er Jahre und ihre relative meinungsbildende Kraft als über die vermeintliche Einheit einer "Schule », die sehr rasch eine starke Differenzierungsfähigkeit bewies. Die Sozialgeschichte, wie sie deren wichtigste Protagonisten zu Beginn der 1970er Jahre definierten, bildete keinen besonderen Bereich der Geschichte, sondern vielmehr eine umfassende Herangehensweise, die politische, wirtschaftliche und kulturelle Phänomene in der Perspektive der Gesellschaft in den Blick nahm ${ }^{9}$. Die gleiche Beobachtung gilt für die 1975 gegründete Zeitschrift Geschichte und Gesellschaft, deren Programm sich stärker durch einen ausgeprägten Sinn für Polemik auszeichnete als durch einen streng soziologischen Zugriff, wie der Untertitel Zeitschrift für historische Sozialwissenschaft nahe zu legen schien. Insgesamt schrieb sich die Erneuerung der 1970er Jahre in ein Referenzfeld mit bisweilen weit entfernten Bezügen ein, wenn man etwa an die ganz allgemein gehaltenen Bezeichnungen der Lehrstühle der Universität Bielefeld denkt, die für viele Beobachter an den 1923 unter der Bezeichnung

9. Significatif est à cet égard le volume collectif édité par WeHLER, 1972, qui couvre tout l'éventail des approches socio-culturelles en incluant, à côté de contributions d'Eric Hobsbawm et Fernand Braudel, des articles de Norbert Elias et Hans Freyer. La contribution de Theodor Schieder est particulièrement intéressante: elle relève de la composante anthropologique d'une approche structurelle déjà longuement discutée dans SCHIEDER, 1965, ici 1968, p. 175 sqq. 
générale de l'histoire » pour Kurt Breysig, qui comme Lamprecht souhaitait rapprocher histoire et sciences exactes ${ }^{10}$.

La véritable controverse qui mérite ce nom s'est développée avec la lente différenciation des approches dans des champs de recherche qui, malgré leur importance, semblaient injustement négligés par une historiographie portée essentiellement sur le développement de la société industrielle. L'enquête la plus stimulante fut dans ce contexte celle sur la production proto-industrielle lancée par un groupe de jeunes chercheurs à l'Institut Max-Planck de Göttingen dont les premiers résultats parurent en $1978^{11}$. Le défi du groupe était de présenter une " théorisation partielle » de la phase de transition d'un type de société à une autre en recourant à une exposition mêlant narration et expression théorique tout en insistant sur l'historicité des caractères systémiques dégagés ${ }^{12}$. En admettant le caractère expérimental de cette approche, qui en visant la société globale s'appuyait essentiellement sur des enquêtes locales, le groupe introduisait explicitement la controverse théorique dans son dispositif heuristique auquel les nombreuses field studies sur des sociétés paysannes partielles servaient de modèles ${ }^{13}$. Stratégies d'héritage et pratiques de procréation, coutumes et représentations religieuses, les instruments d'une démographie du troisième niveau comme ceux

"Theorie der Gesellschaft und Allgemeine Geschichtswissenschaft » für Kurt Breysig, der wie Lamprecht Geschichte und exakte Wissenschaften zusammenführen wollte, geschaffenen Lehrstuhl erinnerte ${ }^{10}$.

Die eigentliche Kontroverse, die wirklich diesen Namen verdient, hat sich mit der langsamen Differenzierung des Herangehensweisen entwickelt in Bereichen der Forschung, die trotz ihrer Bedeutung ungerechterweise vernachlässigt schienen von einer Geschichtsschreibung, die sich im wesentlichen mit dem Aufkommen der Industriegesellschaft beschäftigte. Die anregendste Enquete war in diesem Zusammenhang das Forschungsprojekt über die protoindustrielle Produktion, das eine Gruppe junger Historiker am Göttinger Max-Planck-Institut für Geschichte lancierte und deren erste Ergebnisse 1978 publiziert wurden ${ }^{11}$. Der Anspruch der Gruppe war, eine "partielle Theorie » der Übergangsphase von einem Gesellschaftstyp zu einem anderen zu präsentieren durch eine Darstellungsweise, die Narration und theoretische Erörterung kombinierten, wobei sie stets die Geschichtlichkeit der herausgearbeiteten systemischen Merkmale betonten ${ }^{12}$. Indem sie den experimentellen Charakter dieses Vorgehens, das auf die Gesamtgesellschaft gerichtet war, aber sich im wesentlichen auf Lokalstudien stützte, einräumte, führte die Gruppe ausdrücklich die theoretische Kontroverse in ihr heuristisches Dispositiv ein, für das die zahlreichen field studies über einzelne bäuerliche Gesellschaften als Modelle dienten ${ }^{13}$. Vererbungsstrategien und generatives Verhalten, Bräuche und religiöse Vorstellungen, die Instrumente einer Demographie

10. À propos de Breysig et de la réaction des historiens traditionnels en 1923, voir LePENIES, 1990 , p. 249.

11. Kriedte, Medick et Schlumbohm, 1978

12. Kriedte, Medick et Schlumbohm, 1978, p. 13-35.

13. À commencer par les travaux de Jack Goody, Charles Tilly et David Sabean, voir par exemple Kriedte, Medick et Schlumbohm, 1978, p. 92, n. 7. 
de l'histoire des prix agraires et des marchés locaux devaient se conjuguer dans ce qui se voulait une prise en considération de l'ensemble des facteurs sociaux à l'œuvre dans un processus de transformation sociale.

L'intérêt de la proposition résidait pour une très grande part dans la prise en charge d'une multiplicité d'approches, qui jusqu'ici s'étaient plus ou moins ignorées, et dans leur intégration dans une démarche qui se voulait a priori ouverte. Le fait d'avoir introduit la controverse théorique à l'intérieur même du projet n'était pas sans importance pour l'accueil que lui faisait la communauté scientifique à travers le monde. La multiplication des enquêtes parallèles tout comme les travaux monographiques des auteurs du projet eux mêmes devaient, selon la logique même de l'entreprise, pousser autant à l'affinement des instruments théoriques qu'à l'exploitation de gisements de sources jusqu'ici ignorés.

\section{Discontinuités et échanges triangulaires}

Le regard rétrospectif fait converger des expériences hétérogènes et les condense dans un mouvement plus ou moins spontané que David Sabean caractérise, non sans ironie, de la façon suivante quand il note: "Like Mr Jourdain, who found out that he had been speaking prose for forty years without knowing it, I had been practicing "microhistory" without being aware that it might have a name. I have left it to my former and present colleagues, Hans Medick and Carlo Ginzburg, respectively, to explain the assumption behind the methodology ${ }^{14}$. " De fait, les deux livres de Sabean sur le village de Neckarhausen dans l'Allemagne du Sud-Ouest à l'époque moderne

des dritten Niveaus wie der Methoden der Geschichte der Agrarpreise und der lokalen Märkte sollten sich zu einer Geschichte verbinden, welche die Gesamtheit der sozialen Faktoren berücksichtigen wollte, die in einem Prozeß sozialen Wandels am Werk waren.

Die Bedeutung dieses Vorschlags lag größtenteils darin, daß eine Vielzahl von bis dahin mehr oder weniger ignorierten Ansätzen berücksichtigt und zusammengeführt wurden in einer sich prinzipiell als offen verstehenden Herangehensweise. Für die positive Aufnahme ihres Projekts bei der internationalen Forschergemeinde spielte sicherlich eine nicht unwesentliche Rolle, daß sie die theoretische Kontroverse in den Kern ihres Projekts eingeführt hatten. Die von ihnen angeregten zahlreichen Einzelstudien wie die monographischen Arbeiten der Autoren des Projekts sollten nach der Logik des ganzen Unternehmens die Verfeinerung des theoretischen Instrumentariums gestatten wie die Erschließung bislang unbekannter Quellenschichten.

Ungleichzeitigkeiten und Dreiecksgeschichten

Im Rückblick konvergieren häufig heterogene Forschungserfahren in einer mehr oder weniger spontanen "Bewegung ». David Sabean hat dies mit gewisser Ironie so ausgedrückt: "Like Mr Jourdain, who found out that he had been speaking prose for forty years without knowing it, I had been practicing "microhistory" without being aware that it might have a name. I have left it to my former und present colleagues, Hans Medick and Carlo Ginzburg, respectively, to explain the assumption behind the methodology ${ }^{14}$. »

14. Sabean, 1998, p. Xxiv. 
sont l'exemple emblématique de la rencontre entre l'histoire (comme discipline) et l'anthropologie sociale et culturelle anglo-américaine ${ }^{15}$. Témoin de l'inscription des études historiques dans la tradition des community studies anglaises et de la démographie historique des deux côtés de la Manche, ils apparaissent ex post comme une réalisation poussée du programme de recherche clairement positiviste esquissé par Carlo Poni et Carlo Ginzburg dans leur bref article «Il nome e il come », plaidant pour la reconstruction de la vie des gens à partir du nom, sur la base de toutes les informations accessibles dans les sources ${ }^{16}$.

Le débat épistémologique international des années 1970 devenait ainsi le terrain de rencontre entre la «microstoria » italienne et la «microanalyse » allemande, terme qui aurait été, selon Jürgen Schlumbohm, emprunté à Jacques Dupâquier ${ }^{17}$. Inventés parallèlement pour désigner une alternative à une histoire des structures macrohistoriques ${ }^{18}$, ils s'imposent sans que les différents contextes et adversaires soient toujours clairement présents et pris en considération. On cherche des soutiens et des alliés, des « sœurs ${ }^{19}$ » ou des « frères inconnus ${ }^{20}$ » qu'on découvre par la voie de rencontres triangulaires, avec l'anthropologie sociale britannique ${ }^{21}$ ou encore avec

Und tatsächlich sind die beiden Studien Sabeans über das Dorf Neckarhausen in Südwestdeutschland in der frühen Neuzeit das emblematische Beispiel der Begegnung zwischen der Geschichte (als Fach) und der anglo-amerikanischen Sozial- und Kulturanthropologie ${ }^{15}$. Sie standen in der Tradition der englischen community studies und der historischen Demographie, wie sie aufbeiden Seiten des Ärmelkanals betrieben wurde, erschienen aber im Nachhinein zugleich als eine der weitgehendsten Verwirklichungen des entschieden positivistischen Forschungsprogramms, das Carlo Poni und Carlo Ginzburg 1979 in ihrem kurzen Text "Il nome e il come » skizziert hatten: ein Plädoyer für die Rekonstruktion des Lebens der Menschen ausgehend vom Namen, auf der Grundlage aller in den Quellen verfügbaren Informationen ${ }^{16}$.

Die internationale epistemologische Debatte der 1970er Jahre wurde somit das Terrain einer Begegnung zwischen der italienischer microstoria und der deutschen "Mikro-Analyse » (ein Jürgen Schlumbohm zufolge von Jacques Dupâquier übernommener Ausdruck) ${ }^{17}$. Diese parallel erfundenen Begriffe, um eine Alternative zur makrohistorischen Strukturgeschichte zu bezeichnen ${ }^{18}$, setzten sich durch, ohne daß die unterschiedlichen Bedingungen und Gegner in den verschiedenen Ländern stets präsent und berücksichtigt worden wären. Man suchte Unterstützung und Verbündete, "Schwestern ${ }^{19}$ » und " unbekannte Brüder ${ }^{20} »$, die man durch Dreiecksbegegnungen fand, mit der britischen Sozialanthropologie ${ }^{21}$ oder mit dem History workshop movement, dem Vorbild der deutschen Geschichtswerkstätten.

15. SABEAN, 1990 et 1998.

16. Ginzburg et Poni, 1979. Voir pour la réception française Revel, 1989; Revel, dir., 1996.

17. Sснцимвонм, 1998, p. 25-26.

18. Voir Sснцимвонм, 1998.

19. МеDICK, 1996, p. 40.

20. «Molti fratelli nel mondo » que les microhistoriens italiens « non conoscono ancora », GRENDI, 1994 , p. 539 , n. 4 , p. 548.

21. Voir Gribaudi, 1998. 
le History Workshop Movement qui servira de modèle aux Geschichtswerkstätten en Allemagne.

Le résultat a été des croisements terminologiques étonnants. Ainsi, les historiens italiens qui publient dans les années 1970 une revue d'histoire régionale, les Quaderni storici, voulant se détacher d'une « histoire-synthèse » idéaliste se situant dans la tradition rhétorique, plaident pour une « voie italienne vers l'historiographie sociale plus avancée » ouvrant la voie à une « histoire de la société ${ }^{22}$. À première vue, cela entre étrangement en résonance avec les protagonistes de la historische Sozialwissenschaft et de la Gesellschaftsgeschichte en Allemagne qui devenaient au même moment la cible des partisans allemands d'une approche micro-historique se référant à une anthropologie culturelle herméneutique ${ }^{23}$, dont les dérives culturalistes ont été vigoureusement critiquées par Giovanni Levi et Carlo Ginzburg ${ }^{24}$.

S'il y a un sentiment de symbiose, celui-ci semble plutôt résider dans l'empathie aux connotations éminemment politiques de la Alltagsgeschichte, de l'histoire du monde rural ou de la culture populaire allemande avec les « gens sans nom ${ }^{25}$ » du passé et l'aspiration à saisir la complexité et la plénitude de la vie au niveau « micro » dans les Quaderni storici, et plus généralement le souci de faire entendre les voix authentiques du passé. S'il y avait convergence, elle était surtout due au sentiment de participer à

Das Ergebnis waren erstaunliche terminologische Überschneidungen. So plädierten die italienischen Historiker, die in den 1970er Jahren eine regionalgeschichtliche Zeitschrift namens Quaderni storici herausgaben und sich von der italienischen rhetorischen Tradition der "storia sintesi » abgrenzen wollten, für einen "italienischen Weg zu einer fortgeschritteneren Sozialgeschichte ${ }^{22}$, die den Weg zu einer "Geschichte der Gesellschaft » öffnen sollte. Auf den ersten Blick gibt es hier erstaunliche Übereinstimmungen mit den Protagonisten der historischen Sozialwissenschaft und der Gesellschaftsgeschichte in Deutschland. Diese wurden zur gleichen Zeit die Zielscheibe deutschen Anhänger eines mikrohistorischen Vorgehens, das sich auf eine hermeneutische Kulturanthropologie bezog ${ }^{23}$, deren kulturalistischer Holismus entschieden von Giovanni Levi und Carlo Ginzburg kritisiert wurde ${ }^{24}$.

Wenn es ein Gefühl der Symbiose gab, so lag dies vielleicht eher in der Empathie (mit eminent politischen Implikationen) der Alltagsgeschichte, der Geschichte der bäuerlichen Welt und der Volkskultur in Deutschland mit den "Namenlosen ${ }^{25}$ » der Vergangenheit. Und, bei den Quaderni storici, in dem Bestreben, die Komplexität und Fülle des Lebens auf der Mikroebene zu erfassen, sowie ganz allgemein im Wunsch, die authentischen Stimmen der Vergangenheit hörbar zu machen. Beide Strömungen stimmten sicherlich darin überein, zu einer transnationalen Bewegung zu gehören, die neue Forschungsfelder und neue Fragestellungen eröffnen wollte, wie etwa die Frauen- und Geschlechtergeschichte, sie sich freilich bereits ihre eigenen Foren und

22. Une " "via italiana" verso la storiografia sociale più avanzata" et vers une «storia della società », CARACCIOLO, 1986, p. 618.

23. MEDICK, 1984.

24. LeVI, 1985a.

25. «die Namenlosen », Schlumboнm, 1994, p. 21-30. 
un mouvement visant à ouvrir des nouveaux chantiers comme l'histoire des femmes et la gender history, qui avaient créé leurs propres forums et lieux de publication ${ }^{26}$. Tout compte fait, ce sont les résultats et expériences de recherche, l'évolution thématique et problématiques des revues d'histoire dans les deux pays (Quaderni storici et Historische Anthropologie) et la vulgarisation de la démarche microhistorique qui donnent la mesure de son impact réel ${ }^{27}$.

Les liens méthodologiques explicites entre les deux courants concernent la protoindustrialisation, à commencer avec l'étude d'Albert Schnyder-Burghartz de $1992^{28}$. S'y ajoutaient les résultats de l'enquête du groupe de Göttingen revenant en partie sur les hypothèses initiales en tenant compte des critiques ${ }^{29}$. Se penchant sur la protoindustrie en milieu urbain, Peter Kriedte consacrait ainsi une importante étude à une histoire en mouvement autour de $1848^{30}$. Quant à la référence à la microhistoire, si elle est toujours présente, elle apparait dans des ouvrages récents « d'histoire sociale d'inspiration anthropologique » plus comme un moment de l'enquête que comme une proposition méthodologique forte ${ }^{31}$. Est-ce dû au fait que la réception allemande de la microstoria en Allemagne a été majoritairement culturaliste? Pour s'en convaincre, il suffit de regarder les ouvrages de la collection italienne traduits en allemand (et surtout ceux qui ne l'ont pas été ${ }^{32}$ ) ou de faire le test toujours instructif dans les manuels

Organe schufen ${ }^{26}$. Letztlich läßt sich der Grad an Übereinstimmung und die wirkliche Bedeutung des Ansatzes eher an den Forschungserfahrungen und -ergebnissen ablesen, d.h. an der Entwicklung der Themen und Probleme, die in den jeweiligen Zeitschriften (Quaderni storici und Historische Anthropologie) verhandelt werden, und an der Behandlung der Mikro-Historie in der Handreichungs-Literatur (Einführung, Kompass, Kompendium) ${ }^{27}$.

Explizite methodologische Verbindungen zwischen den beiden Strömungen in Italien und im deutschsprachigen Raum gab es in der Protoindustrialisierungsforschung, beispielsweise mit der Studie von Albert Schnyder-Burghartz aus dem Jahr $1992^{28}$. Und natürlich in den Monographien der Göttinger Gruppe, die teilweise die Ausgangshypothesen zurücknahmen und auf geäußerte Kritik reagierten ${ }^{29}$. Peter Kriedte etwa widmete eine wichtige Untersuchung der städtische Protoindustrie und sozialen Bewegungen in einer Zeit tiefgreifenden Wandels um $1848^{30}$. Der Bezug auf die MikroGeschichte ist in den neueren Untersuchungen " einer anthropologisch orientierten Sozialgeschichte " weiter präsent, aber nur als ein Aspekt der Untersuchung, nicht mehr als starker methodologischer Ansatz ${ }^{31}$. Hängt dies damit zusammen, daß die deutsche Rezeption der microstoria vor allem kulturalistisch war? Um sich davon zu überzeugen, genügt ein Blick darauf, welche Bände der italienischen gleichnamigen Buchreihe ins Deutsche übersetzt worden sind (und vor allem, welche nicht ${ }^{32}$ ), oder

26. Voir par exemple Pomata, 1983, 1991 et 1995.

27. KaISER, 1999; RAULFF, 1996.

28. SCHNYDER-BURGHARTZ, 1992.

29. PonI, 1982, en particulier p. 1105 et 1110 (l'absence de la « proto-industria urbana »).

30. KRIEDTE, 1991, référence à la microhistoire p. 22-23.

31. Quatrième de couverture de SCHINDLER, 2001.

32. KAISER, 1999 et 2000. 
destinés aux étudiants ${ }^{33}$. En tirant la microhistoire presque exclusivement vers les « histoires de vie » qu'on saisirait dans leur plénitude au niveau micro, un ouvrage récent donne un portrait presque caricatural de cette microhistoire ${ }^{34}$.

La présentation d'un canon de lectures et d'auteurs gommant les différences importantes entre eux, est généralement le signe du refroidissement d'une controverse autrefois brûlante. Non seulement, en Allemagne, le débat semble clos, mais on pourrait aussi analyser désormais ce qui s'est passé en termes de sociologie du savoir et retracer ainsi l'institutionnalisation d'une anthropologie historique en Allemagne (ou dans le monde germanophone) ayant désormais ses revues et collections, ses chaires universitaires et ses positions de pouvoir dans les commissions qui pourvoient aux postes universitaires en transformant une position critique en affirmation.

L'anthropologie historique dont l'intention critique était de faire ressortir les logiques spécifiques des " gens sans voix » semble se diluer et donne, avec la différenciation des thèmes, l'impression d'une certaine Gemütlichkeit, d'une sorte de quiétude. Toutefois, on peut se demander s'il ne s'agit pas là plutôt des difficultés intrinsèques d'une anthropologie historique tentée de s'ouvrir aux dimensions profondes de l'existence humaine tout en historisant ses objets et en soulignant leur caractère construit; mais qui, par ailleurs, se confronte toujours à la tradition allemande de l'anthropologie

den immer instruktiven Test zu machen und einen Blick in die Handreichungsliteratur für Studenten zu werfen ${ }^{33}$. Eine gerade erschienene Einführung in die Mikrogeschichte interpretiert diese im wesentlichen als Möglichkeit zur Darstellung von "Lebensgeschichten ", die man auf Mikroniveau in ihrer ganzen Fülle erfassen könne - und gibt damit eine ziemlich verzerrte Darstellung dieser Forschungsrichtung, zumindest in Italien $^{34}$.

Die Präsentation eines Kanons von Texten und Autoren, bei dem die wichtigen Differenzen zwischen diesen in den Hintergrund gedrängt werden, ist im Allgemeinen ein sicheres Anzeichen dafür, daß eine einstmals heftige Debatte sich inzwischen abgekühlt hat. In Deutschland scheint nicht nur die Debatte um die Mikrogeschichte abgeschlossen zu sein, man könnte die Entwicklung mittlerweile wissenschaftssoziologisch analysieren und die Institutionalisierung einer historischen Anthropologie in Deutschland oder im deutschsprachigen Raum nachzeichnen. Diese hat ihre Zeitschriften und Buchreihen, ihre Lehrstühle und Machtpositionen in den Gremien, die über die Besetzung der Positionen entscheiden. Das ist der Lauf der Welt, die Verwandlung einer kritischen Position in eine affirmative.

Die historische Anthropologie, deren kritische Absicht darin lag, die Eigenlogiken der "Menschen ohne Stimme 》 aufzuzeigen, scheint sich thematisch auszufasern und macht den Eindruck einer gewissen Gemütlichkeit. Man mag sich freilich fragen, ob dies nicht auch an den inhärenten Schwierigkeiten einer historischen Anthropologie liegt, die sich den grundlegenden Dimensionen der menschlichen Existenz öffnen, ihre Gegenstände dabei historisieren und deren Natur als Konstrukt unterstreichen will, und

33. Daniel, 2001 ; Eibach et LotTes, dir., 2002.

34. UlbRicht, 2009. 
philosophique et systématique ${ }^{35}$ - et qui semble, d'un autre côté, se dissiper dans un champ culturaliste (Kulturwissenschaften) aux contours incertains et traversé par une succession de turns $^{36}$.

Dans ce contexte on peut sans doute regretter que la réflexion en France sur la microhistoire, publiée en 1996 (Jeux d'échelles), n'ait jamais réussi à traverser le Rhin ${ }^{37}$. Étrange réflexion posthume par ailleurs, puisque l'expérience avait été déclarée close par Edoardo Grendi, sur un ton amer, par Giovanni Levi qui n'en faisait qu'un bilan personnel, et par Carlo Ginzburg qui se disait incapable de le faire ${ }^{38}$. Débat intéressant puisque les problèmes épistémologiques furent discutés dans l'horizon d'expériences de recherche très différentes, situées à d'autres niveaux et faisant de la variation un instrument heuristique : le passage de la ville au réseau des villes dans la France moderne, opéré par Bernard Lepetit, en est une démonstration qui donne un autre sens à l'expérimentation en histoire ${ }^{39}$. Autre exemple, l'ouvrage de Giovanni Levi, L'Eredità materiale, dont le héros est, dans la réception allemande, placé à côté du meunier Menocchio de Carlo Ginzburg ${ }^{40}$. La proposition de Levi est autrement prise au sérieux par Bernard Lepetit.

zugleich mit der massiven deutschen Tradition der philosophischen Anthropologie ${ }^{35}$ konfrontiert ist. Und die zugleich in einem Feld namens "Historische Kulturwissenschaft » aufzugehen scheint, einem Ensemble mit unklaren Konturen und geprägt von einer immer rascheren Abfolge von turns ${ }^{36}$.

In diesem Zusammenhang ist es sicherlich zu bedauern, daß die französische Reflexion über die Mikrohistoire (publiziert 1996) es nicht geschafft hat, den Rhein zu überqueren ${ }^{37}$. Eine merkwürdige gleichsam postume Reflexion übrigens, denn das Abenteuer der microstoria war zu diesem Zeitpunkt schon für beendet erklärt worden von Edoardo Grendi (mit einem bitteren Unterton), von Giovanni Levi, der nur eine persönliche Bilanz gezogen hatte, und von Carlo Ginzburg, der sich zu einer solchen persönlichen Bilanzziehung außerstande sah. Eine interessante französische Debatte, denn sie wurde im Horizont ganz anderer Forschungserfahrungen geführt, die auf anderen als der Mikroebene angesiedelt waren und die aus dem Variieren der Niveaus ein heuristisches Instrument gemacht hatten ${ }^{38}$. Bernard Lepetits Wechsel von der Stadt auf die Ebene des französischen Städtenetzes, um den historischen Wandel vom 18. zum 19. Jahrhunderts zu analysieren, ist eine methodologische Demonstration, die auch dem Begriff des Experiments in der Geschichte einen anderen Sinn gibt ${ }^{39}$. Ein anderes Beispiel ist Giovanni Levis Studie, L'Eredità materiale, dessen Held in der deutschen Rezeption dem Müller Menocchio Carlo Ginzburgs zur Seite gestellt wird ${ }^{40}$.

35. Voir la discussion lucide chez TANNER, 2004.

36. Dans EiвAсн et LotтEs, dir., 2002, la microhistoire est intégrée dans une partie appelée « Nouvelle histoire culturelle ». Voir pour une tentative de sauver la microhistoire de la vague culturaliste MEDICK, 1991, et les remarques critiques plaidant pour une anthropologie systématique de SoFSKY, 1991.

37. Revel, dir., 1996. L'ouvrage, préparé par Hans Medick, fut la victime d'une forte réduction de la place des sciences humaines et sociales chez l'éditeur pressenti.

38. Grendi, 1994; Levi, 1991; GinZBURG, 1994.

39. LEPETIT, 1988 et 1996.

40. UlBRicht, 2009. 
Il analyse en profondeur les apories du modèle proposé qui considère l'expérience des acteurs historiques comme la dernière instance sanctionnant le modèle faisant de la distance sociale le facteur explicatif des prix différenciés de la terre ${ }^{41}$.

Un des résultats collatéraux de la reconstruction de la démarche microhistorique a été la découverte du livre de Siegfried Kracauer, History - The Last Things Before the Last (publié posthume en $1969^{42}$ ) dans lequel l'auteur plaide pour la variation des perspectives et des niveaux, une réflexion qu'on pourrait faire remonter dans la tradition allemande jusqu'à l'Allgemeine Geschichtswissenschaft de Johann Martin Chladenius $(1752)^{43}$. Le débat épistémologique se place ainsi sur un terrain plus vaste: 1'aspiration microhistorique de faire de l' « histoire locale comme histoire générale ${ }^{44} »$ n'est qu'une position emphatique dans le débat séculaire sur le rapport entre histoire particulière et histoire générale. Le spatial turn a donné lieu à toute une série de recherches qui ont pris comme point de départ la pluralité et le caractère construit des espaces sociaux des acteurs et la pluralité et la simultanéité des perspectives ${ }^{45}$, ce qui a permis une autre rencontre avec l'expérience de la microstoria ${ }^{46}$.

Le couple histoire particulière/histoire générale rappelle cependant un autre débat récurrent, celui sur le rapport entre histoire particulière et histoire universelle, dont

Ganz anders ernst genommen wird die Proposition Levis von Bernard Lepetit der die Aporien eines Konzepts befrage, das die Erfahrung der historischen Akteure zur letzten Instanz für die Gültigkeit eines Modells erklärt, welches die soziale Distanz zum erklärenden Faktor der Ausdifferenzierung der Bodenpreise macht ${ }^{41}$.

Ein Nebenprodukt der Rekonstruktion des mikrohistorischen Vorgehens war die "Entdeckung» des postum 1969 veröffentlichen Werks von Siegfried Kracauer, History - The Last Things Before the Last ${ }^{42}$, in dem Kracauer für die Variierung der Perspektiven und Analyseebenen plädiert, eine Reflexion, die in der deutschen Tradition bis auf die Allgemeine Geschichtswissenschaft von Johann Martin Chladenius (1752) ${ }^{43}$ zurückgeführt werden kann. Die epistemologische Debatte wird damit auf ein allgemeineres Niveau geführt: die mikrohistorische Absicht, "Lokalgeschichte als Allgemeine Geschichte 44 " zu betreiben, ist nur eine emphatische Position in der jahrhundertealten Debatte über das Verhältnis von partikularer und allgemeiner Geschichte. Der spatial turn hat beispielsweise eine ganze Reihe von Untersuchungen auf den Weg gebracht, die von der Vielfältigkeit möglicher Perspektiven ${ }^{45}$, von der Pluralität und dem konstruierten Charakter der sozialen Räume der Akteure ausgehen und auf diesem Wege ein Terrain der Begegnung mit den Erfahrungen der microstoria gefunden haben ${ }^{46}$.

Das Begriffspaar partikulare und allgemeine Geschichte ruft eine andere wiederkehrende Debatte ins Gedächtnis, diejenige über das Verhältnis von partikularer und

41. Levi, 1985b, chap. III. Olmo et Lepetit, 1995, p. 15-16; LePETit, 1996, p. 81.

42. Kracauer mourut en 1966. Kracauer, 1969. Discussion chez Medick, 1994, 1996; Ginzburg, 1994; SChlumbohm, 1998.

43. Chladenius, 1985.

44. C'est le sous-titre du livre de Medick, 1996.

45. Voir les contributions dans DürR et Schwerhoff, éd., 2005.

46. Voir la contribution d'Angelo Torre dans Rau et Schwerhoff, dir., 2008; TorRe, 2006. 
les moments forts autour de 1800 et de 1900 reflètent des crises profondes du monde occidental, aux connotations politiques mais aussi aux conséquences épistémologiques évidentes ${ }^{47}$. Nous y sommes revenus depuis un moment déjà. Les notions comme « entangled» ou «connected histories », ou encore " histoire croisée », proposées dans le champ de la global history ${ }^{48}$, essayent de saisir les articulations entre différentes expériences historiques. Que signifient la multiperspectivité (et la nécessaire et heuristiquement positive - sélection de perspectives ${ }^{49}$ ) ou la variation des niveaux d'analyse dans ce champ ${ }^{50}$ ? Pour éviter le remake d'un débat clos, il faudra réfléchir sur ces questions historiographiques et épistémologiques dans cette perspective et dans l'horizon des problèmes du monde actuel.

Universalhistorie, deren Höhepunkte um 1800 und 1900 tiefgreifende Krisen der europäischen und westlichen Welt reflektieren, mit politischen und epistemologischen Implikationen ${ }^{47}$. Seit einiger Zeit schon befinden wir uns erneut in einer derartigen, historischen neuartigen Situation. Begriffe wie " entangled ", " connected histories " oder " histoire croisée ", die in der global history ${ }^{48}$ diskutiert werden, versuchen die Verknüpfungen zwischen verschiedenen historischen Erfahrungen zu erfassen. Was bedeuten hier Multiperspektivität (und die notwendige und heuristisch positive Auswahl von Perspektiven ${ }^{49}$ ) oder die Variierung von Analyse-Ebenen ${ }^{50}$ ? Wenn wir ein remake einer abgeschlossenen Debatte vermeiden möchten, sollten wir über diese historiographischen und epistemologischen Fragen im Horizont der gegenwärtigen Welt diskutieren.

\section{LISTE DES RÉFÉRENCES}

Aslanian (Sebouh), 2007, «From the Indian Ocean to the Mediterranean: Circulation and the Global Trade Networks of Armenian Merchants from New Julfy, 1605-1748 », thèse de doctorat dactylogr., Columbia University.

Bergenthum (Hartmut), 2004, Weltgeschichten im Zeitalter der Weltpolitik, Munich, Meidenbauer.

CARACCIOlo (Alberto), 1986, « Gli Indici di "Quaderni storici”: una rivista modernistica? », Quaderni storici, $\mathrm{n}^{\circ}$ 62, p. 613-620.

Chladenius (Johann Martin), 1985, Allgemeine Geschichtswissenschaft, réimpr. de l'édition de Leipzig, 1752, introd. Christoph Friedrich, préf. Reinhart Koselleck, Vienne, Böhlau.

Conrad (Christoph), 2006, « Die Dynamik der Wenden. Von der neuen Sozialgeschichte zum cultural turn », dans Osterhammel et al., éd., 2006, p. 133-160.

Daniel (Ute), 2001, Kompendium Kulturgeschichte. Theorien, Praxis, Schlüsselwörter, Francfort-sur-le-Main, Suhrkamp.

47. Escudier, 2003 ; Bergenthum, 2004.

48. Voir la discussion dans Temps croisés, mondes mêlés, 2001, et Histoire globale, histoires connectées, 2007.

49. Voir la préface de Reinhart Koselleck à Chladenius, 1985, p. VII-IX.

50. Pour l'articulation de niveaux et d'angles de recherche dans un horizon global voir TRIVELLATO, 2009; AsLanian, 2007; et déjà LomBARd et AuBin, éd., 1988. Voir aussi les remarques méthodologiques dans l'introduction à Osterhammel, 2009, p. 13-21. 
DÜrR (Renate) et Schwerhoff (Gerd), éd., 2005, « Kirchen, Märkte und Tavernen. Erfahrungsund Handlungsräume in der Frühen Neuzeit », Zeitsprünge, n ${ }^{\circ}$ 9, p. 3-4.

Eibach (Joachim) et LotTes (Günther), dir., 2002, Kompass der Geschichtswissenschaft, $2^{\mathrm{e}}$ éd., Göttingen, Vandenhoeck \& Ruprecht, 2006.

Escudier (Alexandre), 2003, « De Chladenius à Droysen. Théorie et méthodologie de l'histoire de langue allemande (1750-1860) », Annales. Histoire, sciences sociales, ${ }^{\circ} 4$, p. 743-777.

GinzBurg (Carlo), 1994, «Microstoria: due o tre cose che so di lei », Quaderni storici, ${ }^{\circ}$ 86, p. 510-539; "Mikro-Historie. Zwei oder drei Dinge, die ich von ihr weiß», Historische Anthropologie, vol. I, n 2, 1993, p. 169-192.

GinzBurg (Carlo) et Poni (Carlo), 1979, «Il nome e il come. Mercato storiografico e scambio inuguale », Quaderni storici, ${ }^{\circ} 49$, p. 1-17; «La microhistoire», trad. franç. partielle, Le Débat, $\mathrm{n}^{\circ} 17,1981$, p. 133-136; «Was ist Mikrogeschichte?», trad. all. partielle, Geschichtswerkstatt, $\mathrm{n}^{\circ}$ 6, 1985, p. 48-52.

Grendi (Edoardo), 1994, «Ripensare la microstoria? », Quaderni storici, n 86, p. 539-549; « Repenser la microhistoire? », dans ReVEL, dir., 1996, p. 233-243.

Gribaudi (Maurizio), 1998, «Avant-Propos », dans ID., dir., Espaces, temporalités, stratifications. Exercices sur les réseaux sociaux, Paris, Éditions de l'École des hautes études en sciences sociales, p. 5-40.

Histoire globale, histoires connectées, 2007, nº spéc. de la Revue d'Histoire moderne et contemporaine, $\mathrm{n}^{\circ} 5$.

KAISER (Wolfgang), 1999, «Fratelli sconosciuti e una sorella lontana. I Quaderni storici in una prospettiva esterna $»$, Quaderni storici, $\mathrm{n}^{\circ} 100$, p. 31-48.

KAISER (W.), 2000, «Regionalgeschichte, Mikro-Historie und segmentierte Öffentlichkeiten. Ein vergleichender Blick auf die Methodendiskussion », dans BraKensIEK (Stefan) et FLÜGEL (Axel), éd., Regionalgeschichte in Europa. Methoden und Erträge der Forschung vom 16. bis zum 19. Jahrhundert, Paderborn, Schöningh, p. 25-44.

Kocka (Jürgen), 2002, Sozialgeschichte in Deutschland. Aufstieg - Krise - Perspektiven, Brunswick/Bonn, Historisches Forschungszentrum.

Kracauer (Siegfried), 1969, History - The Last Things Before the Last, New York, Oxford University Press; Geschichte - Vor den letzten Dingen, trad. all. Karsten Wiтte, Francfortsur-le-Main, Suhrkamp (Schriften, 4), 1971; L'Histoire des avant-dernières choses, trad. franç. Claude Orsoni, prés. Jacques Revel, Paris, Stock, 2006.

Kriedte (Peter), 1991, Eine Stadt am seidenen Faden. Haushalt, Hausindustrie und soziale Bewegung in Krefeld in der Mitte des 19. Jahrhunderts, Göttingen, Vandenhoeck \& Ruprecht.

Kriedte (Peter), Medick (Hans) et Schlumbohm (Jürgen), 1978, Industrialisierung vor der Industrialisierung. Gewerbliche Warenproduktion auf dem Land in der Formationsperiode des Kapitalismus, mit Beiträgen von Herbert Kisch und Franklin F. Mendels, Göttingen, Vandenhoeck \& Ruprecht.

LEPENIES (Wolf), 1990, «Disciplines concurrentes: sociologie et historiographie », dans ID., Les Trois Cultures. Entre science et littérature l'avènement de la sociologie, Paris, Éditions de la Maison des sciences de l'homme.

LePETIT (Bernard), 1988, Les Villes dans la France moderne (1740-1840), Paris, Albin Michel.

Lepetit (B.), 1996, « De l'échelle en histoire », dans Revel, dir., 1996, p. 71-94.

Levi (Giovanni), 1985a, « I pericoli del geertzismo », Quaderni storici, n 58, p. 269-277.

Levi (G.), 1985b, L'Eredità immateriale. Carriera di un esorcista nel Piemonte del Seicento, Turin, Einaudi; Le Pouvoir au village. Histoire d'un exorciste dans le Piémont du XVII siècle, trad. franç. Monique AyMARD, Paris, Gallimard, 1989. 
Levi (G.), 1991, « On Microhistory », dans BuRke (Peter), éd., New Perspectives on historical writing, Oxford, Oxford University Press, p. 93-113.

Lombard (Denys) et AuBIN (Jean), éd., 1988, Marchands et hommes d'affaires asiatiques dans l'océan Indien et la mer de Chine, XIII ${ }^{e}-X X^{e}$ siècles, Paris, Éditions de l'École des hautes études en sciences sociales.

Medick (Hans), 1984, « Missionare im Ruderboot? Ethnologische Erkenntnisweisen als Herausforderung an die Sozialgeschichte », Geschichte und Gesellschaft, ${ }^{\circ}$ 1, p. 325-343.

Medick (H.), 1991, «Quo vadis Historische Anthropologie? Geschichtsforschung zwischen Historischer Kulturwissenschaft und Mikro-Historie », Historische Anthropologie, vol. IX, $\mathrm{n}^{\circ} 1$, p. 78-92.

Medick (H.), 1994, « Mikro-Historie », dans Schulze (Winfried), dir., Sozialgeschichte, Alltagsgeschichte, Mikro-Historie. Eine Diskussion, Göttingen, Wallstein, p. 40-53.

MEDICK (H.), 1996, Weben und Überleben in Laichingen, 1650-1900. Lokalgeschichte als Allgemeine Geschichte, Göttingen, Vandenhoeck \& Ruprecht.

Olmo (Carlo) et Lepetit (Bernard), 1995, «E se Erodoto tornasse in Atene? Un possibile programma di storia urbana per la città moderna », dans ID., dir., La Città e le sue storie, Turin, Einaudi, p. 3-50.

Osterhammel (Jürgen) et al., éd., 2006, Wege der Gesellschaftsgeschichte, Göttingen, Vandenhoeck \& Ruprecht.

Osterhammel (J.), 2009, Die Verwandlung der Welt. Eine Geschichte des 19. Jahrhunderts, $3^{\mathrm{e}}$ éd., Munich, Beck.

Pomata (Gianna), 1983, «Die Geschichte der Frauen zwischen Anthropologie und Biologie », Feministische Studien, ${ }^{\circ}$ 2, p. 113-127.

Pomata (G.), 1991, « Partikulargeschichte und Universalgeschichte - Bemerkungen zu einigen Handbüchern der Frauengeschichte », L'Homme. Zeitschrift für feministische Geschichtswissenschaft, $\mathrm{n}^{\circ} 2$, p. 5-44.

Pомата (G.), 1995, « Vollkommen oder verdorben? Der männliche Samen im frühneuzeitlichen Europa », L'Homme. Zeitschrift für feministische Geschichtswissenschaft, n ${ }^{\circ} 6 / 2$, p. 59-85.

Poni (Carlo), 1982, «Protoindustrializzazione: un commento», Quaderni storici, $\mathrm{n}^{\circ}$ 51, p. 1103-1111.

Rau (Susanne) et Schwerhoff (Gerd), dir., 2008, Topographien des Sakralen. Religion und Raumordnung in der Vormoderne, Munich-Hambourg, Dölling und Galitz Verlag.

RaulfF (Ulrich), 1996, " "Historische Anthropologie". Ein Programm und eine Zeitschrift », Rechtshistorisches Journal, ${ }^{\circ}$ 15, p. 65-79.

Revel (Jacques), 1989, "L'histoire au ras du sol», préface à Levi (Giovanni ), Le Pouvoir au village. Histoire d'un exorciste dans le Piémont du XVII siècle, Paris, Gallimard, p. I-XXXIII.

Revel (J.), dir., 1996, Jeux d'échelles. La microanalyse à l'expérience, Paris, Gallimard/Le Seuil.

Revel (J.), dir., 2006, Giochi di scala. La microstoria alla prova dell'esperienza, Rome, Viella.

Sabean (David), 1990, Property, Production and Family in Neckarhausen, 1700-1870, Cambridge, Cambridge University Press.

Sabean (D.), 1998, Kinship in Neckarhausen 1700-1870, Cambridge, Cambridge University Press.

SCHIEDER (Theodor), 1965, Geschichte als Wissenschaft. Eine Einführung, $2^{\mathrm{e} e}$ éd., Munich, Oldenbourg, 1968.

SCHINDLER (Norbert), 2001, Wilderer im Zeitalter der Französischen Revolution. Ein Kapitel alpiner Sozialgeschichte, Munich, Beck. 
Schlumbонм (Jürgen), 1994, Lebensläufe, Familien, Höfe. Die Bauern und Heuerleute des Osnabrückischen Kirchspiels Belm in proto-industrieller Zeit, 1650-1860, Göttingen, Vandenhoeck \& Ruprecht.

Schlumboнm (J.), 1998, « Mikrogeschichte-Makrogeschichte: Zur Eröffnung einer Debatte », dans ID., éd., Mikrogeschichte-Makrogeschichte: komplementär oder inkommensurabel. Mit Beiträgen von Maurizio Gribaudi, Giovanni Levi und Charles Tilly, Göttingen, Wallstein, p. 9-32.

SCHNYDER-BURGHARTZ (Albert), 1992, Alltag und Lebensformen auf der Basler Landschaft um 1700. Vorindustrielle, ländliche Kultur und Gesellschaft aus mikrohistorischer PerspektiveBretzwil und das obere Waldenburger Amt von 1690 bis 1750, Liestal, Verlag des Kantons Basel-Landschaft.

Schulze (Winfried), 1989, Deutsche Geschichtswissenschaft nach 1945, Munich, Oldenbourg (Beiheft 10 der Historischen Zeitschrift).

Schulze (Winfried) et Oexle (Otto-Gerhard), éd., 1999, Deutsche Historiker im Nationalsozialismus, Francfort-sur-le-Main, Fischer Taschenbuch-Verlag.

SOFSKY (Wolfgang), 1991, «Systematische und historische Anthropologie. Adnoten zu Hans Medicks "Quo vadis Historische Anthropologie" ", Historische Anthropologie, vol. IX, n 3, p. 457-461.

TanNer (Jakob), 2004, Historische Anthropologie zur Einführung, 2éd., Hambourg, Junius, 2008.

Temps croisés, mondes mêlés, 2001, $\mathrm{n}^{\circ}$ spéc. des Annales. Histoire, sciences sociales, $\mathrm{n}^{\circ} 1$.

Theorie der Geschichte, 1977-1990, Munich, Deutscher Taschenburch-Verlag, 6 vol.

Torre (Angelo), 2006, « I luoghi dell'azione », dans Revel, dir., 2006, p. 301-317.

Trivellato (Francesca), 2009, The Familiarity of Strangers. The Sephardic Diaspora, Livorno and Cross-Cultural Trade in the Early Modern Period, New Haven/Londres, Yale University Press.

Ulbricht (Otto), 2009, Mikrogeschichte. Menschen und Konflikte in der Frühen Neuzeit, Francfort-sur-le-Main, Campus.

Wehler (Hans-Ulrich), 1972, Geschichte und Soziologie, Köln, Kiepenheuer \& Witsch (Neue Wissenschaftliche Bibliothek).

Wehler (H.-U.), 1997, «Kommentar », dans Mergel (Thomas) et Welskopp (Thomas), éd., Geschichte zwischen Kultur und Gesellschaft. Beiträge zur Theoriedebatte, Munich, Beck, p. 351-366. 